\title{
As limitações do modelo do campo elétrico do condutor e suas implicações didáticas
}

The limitations of the electric-field model of the conductor and its didactical implications

\author{
K. Slodkowski ${ }^{1}$, M. C. Pinheiro*1, T. C. Luchese ${ }^{1}$ \\ ${ }^{1}$ Universidade Federal da Fronteira Sul, Campus Cerro Largo, Cerro Largo, RS, Brasil
}

Recebido em 12 de Maio, 2017. Revisado em 15 de Setembro, 2017. Aceito em 11 de Outubro, 2017.

\begin{abstract}
Abordamos um clássico problema de Eletrostática presente em livros didáticos do Ensino Médio e Superior: o campo elétrico gerado por um excesso de cargas em uma esfera condutora. De início, analisamos especificamente o valor do campo na superfície da esfera condutora, assumindo que as cargas estão todas distribuídas exatamente nessa superfície. Em seguida, ao flexibilizar a distribuição de cargas para um modelo de distribuição uniforme numa camada esférica de pequena espessura, mostramos que as aparentes discordâncias entre os resultados apresentados na literatura não são matemáticas. Elas apenas evidenciam as limitações dos modelos adotados para representar o objeto.
\end{abstract}

Palavras-chave: modelização, campo elétrico, descontinuidade.

\begin{abstract}
We study a classical electrostatic problem presented in highschool and university textbooks: the electric field generated by a charge excess in a spheric conductor. Firstly, we verify the field value on the surface of the conductor, assuming the singular charge distribution model. Secondly, given some flexibility to the charge distribution to a uniform density over a tiny spherical shell, we show that apparent discrepancies that are presented in literature only give evidence of the limitations of the singular model on describing of the real object.
\end{abstract}

Keywords: modelling process, electric field, discontinuity.

\section{Introdução}

O Ensino e a própria Pesquisa em Física se apoiam em modelos que visam representar objetos reais. Evidentemente, pela complexidade da realidade, essa representação tornase parcial e aproximativa, apresentando apenas aspectos relevantes (definidos pelo pesquisador) do objeto real. Assim, um mesmo objeto pode receber incontáveis modelos que, a depender da profundidade da análise, podem ser divergentes entre si [1]. Com isso em mente, uma abordagem dos conteúdos em sala de aula a partir da modelização pode aproximar o conhecimento cientifico da realidade dos estudantes. Essa análise detalhada das limitações do modelo científico é o norte da discussão que aqui faremos em torno do problema do campo elétrico devido um condutor carregado - problema clássico da Eletrostática -, partindo da controvérsia encontrada entre os trabalhos de Salvatore e Assad, ambos publicados na Revista Brasileira de Ensino de Física [2,3].

Dada uma esfera condutora de raio $R$ e carga $Q$, no vácuo, mostra-se facilmente [4,5] que o campo elétrico a uma distância $r \neq R$ de seu centro tem módulo dado por

$$
E(r)=\left\{\begin{array}{ccc}
0 & \text { para } & r<R \\
\frac{1}{4 \pi \varepsilon_{\circ}} \frac{Q}{r^{2}} & \text { para } & r>R
\end{array}\right.
$$

*Endereço de correspondência: pinheiro.marcio@gmail.com
Tomando os limites laterais em torno de $r=R$, uma descontinuidade em $E(r)$ se estabelece:

$$
\lim _{r \rightarrow R^{-}} E(r)=0 \text { e que } \lim _{r \rightarrow R^{+}} E(r)=\frac{\sigma}{\varepsilon_{0}},
$$

com $\sigma \equiv Q / 4 \pi R^{2}$, a densidade superficial de cargas. A análise dessa descontinuidade não é única nos principais livros didáticos usados no Ensino Médio e no Ensino Superior e é o centro das discussões levantadas por [2, 3 .

Em 6 8], textos usados no Ensino Médio, e também nos clássicos $\left[\begin{array}{l|l|l}4 & 5 & 9\end{array}\right]$ menciona-se brevemente que o campo é diferente de zero nessa região, sem quantificá-lo. Por outro lado, [10 descreve que a intensidade do campo elétrico em pontos da superfície do condutor esférico é $E(R)=\frac{\sigma}{2 \varepsilon_{\circ}}$, isto é, exatamente a média entre os valores limites apresentados em (2), sem, no entanto, apresentar cálculos ou argumentos detalhados. Este valor é o mesmo demonstrado por [2], mas questionado por [3], sob o argumento de que a descontinuidade do campo na superfície do condutor não admite um único valor de campo.

Neste texto, pretendemos mostrar que o resultado de [2] é matematicamente consistente, demonstrando-o de outra forma na Seç. 2, mas que a descontinuidade não passa de uma consequência direta da limitação do modelo do campo elétrico do condutor. Nesse sentido, mostraremos na Seç. 3 que qualquer modelo sutilmente mais elaborado para a distribuição de cargas no condu- 
tor, desde que elimine a singularidade, leva ao mesmo resultado matemático demonstrado na Seç. 2

\section{Campo $\vec{E}$ na superfície do condutor}

O excesso de cargas num condutor se acumula em uma região de aproximadamente $2 \AA$ em torno da última camada atômica que compõe o material 911 . Nesta seção, adotaremos o modelo tradicional, no qual se assume que esse excesso encontra-se exatamente na sua superfície, criando ali uma singularidade. A partir da lei de Coulomb, mostraremos que a intensidade do campo elétrico $E$ na superfície, citado por [10] e demonstrado por 2 ] a partir da Lei de Gauss, é encontrado como um limite desse modelo, mas não implica que a função $E(r)$ seja contínua nesses pontos.

Sabendo que o módulo do campo de um anel de cargas de raio $r$ e carga $q$ em um ponto situado sobre seu eixo de simetria (eixo perpendicular ao plano que contém o anel e passando pelo seu centro) a uma distância $z$ do centro do anel é dado por

$$
E=\frac{1}{4 \pi \epsilon_{0}} \frac{q z}{\left(z^{2}+r^{2}\right)^{3 / 2}},
$$

com direção suportada pelo eixo de simetria e apontando para longe (perto) do anel caso a carga $q$ seja positiva (negativa), obteremos o campo em um ponto $\mathrm{P}$ sobre a superfície da esfera condutora (em $r=R$ ) a partir de uma superposição do campo de vários anéis que comporão a distribuição de cargas. A lógica subjacente para tal feito será a seguinte: assume-se que a esfera condutora adquire a carga $Q$ e então fixam-se estas cargas no espaço de modo a manter a densidade superficial de cargas imutável; em seguida, recorta-se uma calota desta esfera no entorno do ponto $\mathrm{P}$, retirando juntamente com a calota sua carga. Uma vez que as cargas ficaram fixas, a densidade superficial de cargas ficará estável e, uma vez que não há cargas em $\mathrm{P}$, está-se calculando o campo elétrico naquele ponto e não a força sobre uma determinada carga de prova ali colocada.

De acordo com a Fig. 1] vemos que o campo resultante em $\mathrm{P}$ será a soma dos campos $d E$ dos anéis com carga $d Q=2 \pi \sigma R^{2} \operatorname{sen} \theta d \theta\left(\sigma \equiv Q / 4 \pi R^{2}\right)$ e raio $r=R \operatorname{sen} \theta$. A distância do centro de cada anel até o ponto $\mathrm{P}$ será

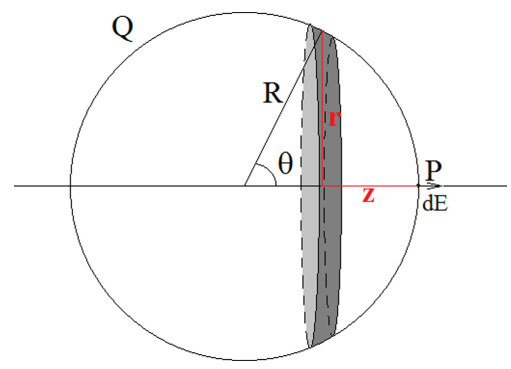

Figura 1: Definição das grandezas associadas ao cálculo do campo no ponto $\mathrm{P}$, sobre a superfície da esfera condutora.

$$
\begin{aligned}
z=R(1-\cos \theta) & \text {. Com essas informações, escreve-se } \\
E & =\int \frac{1}{4 \pi \epsilon_{0}} \frac{d Q z}{\left(z^{2}+r^{2}\right)^{3 / 2}} \\
& =\frac{1}{4 \pi \epsilon_{0}} \int_{\theta_{0}}^{\pi} \frac{2 \pi \sigma R^{3} \operatorname{sen} \theta(1-\cos \theta) d \theta}{\left[2 R^{2}(1-\cos \theta)\right]^{3 / 2}} \\
E & =\frac{\sigma}{2 \epsilon_{0}}\left(1-\sqrt{\frac{1-\cos \theta_{0}}{2}}\right)
\end{aligned}
$$

sendo $\theta_{0}$ o ângulo que define a calota recortada da esfera em torno do ponto P. O limite em que $\theta_{0} \rightarrow 0$ leva ao resultado apresentado por [2,10]:

$$
\lim _{\theta_{0} \rightarrow 0} E=\frac{\sigma}{2 \epsilon_{0}} \text {. }
$$

Vale lembrar que o resultado expresso na equação (5) é o valor do campo resultante em $r=R$ devido a uma distribuição singular de cargas, ou seja, é o campo em $r=R$, consistente como modelo singular adotado, e ainda sem o $d q$ dali retirado com a calota infinitesimal. Desse modo, tem que se ter clareza que, nessa situação, os campos em $r \rightarrow R^{-}$e em $r \rightarrow R^{+}$não serão nulos 1

Os resultados (1), (2) e (5) explicitam a descontinuidade do campo em $r=R$. Da mesma forma, embora tenhamos encontrado um valor limite para o campo $\vec{E}$ num ponto $P$ na casca (Fig. 2a), observa-se que o potencial elétrico não é diferenciável neste ponto (Fig. $2 \mathrm{p}$ ), conforme se espera para qualquer campo conservativo. Tal inconsistência não deve surpreender, uma vez que ela ocorre justamente na região do espaço onde o modelo simplifica a realidade.

Na próxima seção, mostraremos que tanto a descontinuidade do campo quanto o potencial não diferenciável são consequências diretas da singularidade assumida na distribuição de carga.

\section{Casca esférica de espessura $2 \delta$}

De acordo com [3], o campo elétrico não é definido na superfície de um condutor esférico carregado. Por outro lado, é exatamente nessa região que o excesso de carga se encontra, mais precisamente em uma ou duas camadas atômicas da superfície (camada de transição) [5, 9]. Apesar do campo elétrico ser bastante complexo nessa região devido a real distribuição das cargas 11], propomos aqui uma análise de um modelo bastante simples, mas que ainda assim descreve muito bem o objeto, sem recair na tradicional singularidade na distribuição de carga e, consequentemente, numa descontinuidade artificial do campo.

Imaginemos que as cargas se distribuem uniformemente em uma camada muito fina de espessura $2 \delta(\delta \sim 1 \AA)$, formando uma nuvem de densidade uniforme em torno do raio $R$ da esfera. O campo gerado por este objeto

\footnotetext{
$\overline{{ }^{1} \text { Veja mais detalhes em }[9]}$.
} 

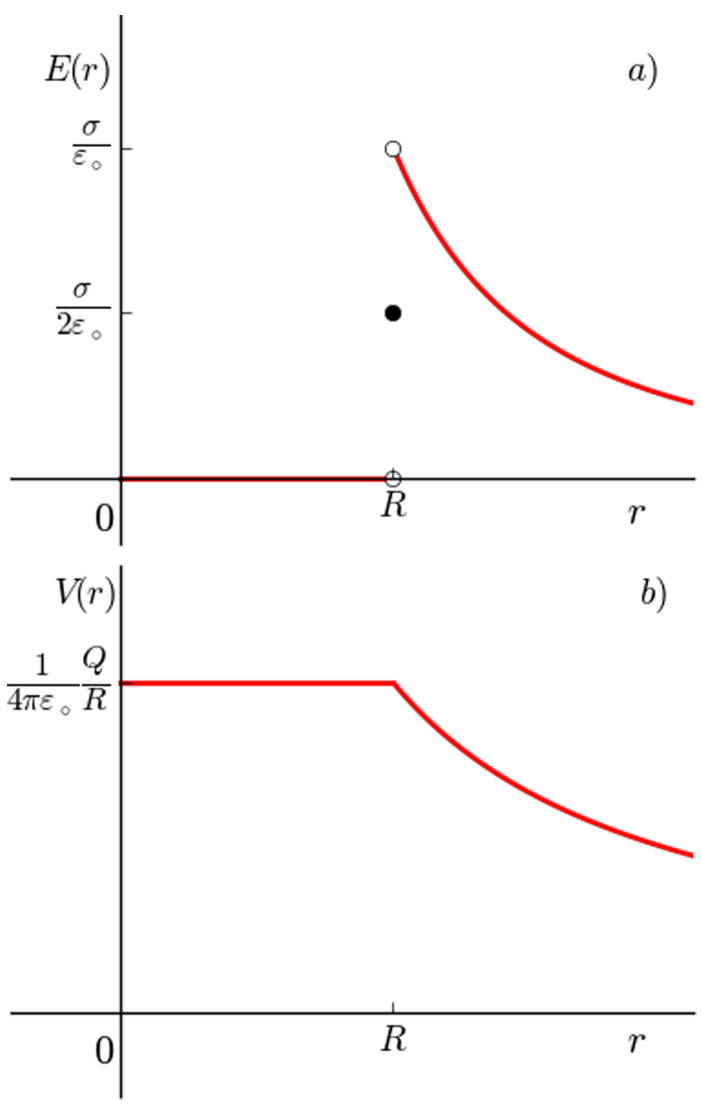

Figura 2: (a) Campo e (b) potencial elétrico devido a uma esfera condutora de raio $R$, como função da distância $r$ com relação ao seu centro, desprezando-se a espessura da região onde se concentra o excesso de cargas.

é análogo ao campo devido a uma casca esférica nãocondutora, de modo que, em ambos os casos, pode ser determinado facilmente a partir da lei de Gauss. Nestas bases, se $R_{1}=R-\delta$ e $R_{2}=R+\delta$ são respectivamente os raios interno e externo da nuvem de cargas e desejamos encontrar o campo em um certo raio $r$, com $R_{1}<r<R_{2}$, definimos uma superfície gaussiana de raio $r$, concêntrica à esfera, de tal modo que

$$
\varepsilon_{\circ} \oint \vec{E}(r) \cdot d \vec{A}=q_{\mathrm{env}} .
$$

Como, por simetria, $\vec{E}$ é radial e, naturalmente, a fração da carga externa a $r$ não contribui para $\vec{E}(r)$, o módulo de $\vec{E}$ se resume a

$$
E(r)=\frac{1}{4 \pi \varepsilon_{\circ}} \frac{q_{\mathrm{env}}}{r^{2}} .
$$

Observando que

$$
q_{\mathrm{env}}=Q \frac{\left(r^{3}-R_{1}^{3}\right)}{\left(R_{2}^{3}-R_{1}^{3}\right)},
$$

obtemos

$$
E(r)=\frac{Q}{4 \pi \varepsilon_{\circ}} \frac{r}{R_{2}^{3}} \frac{\left(1-\frac{R_{1}^{3}}{r^{3}}\right)}{\left(1-\frac{R_{1}^{3}}{R_{2}^{3}}\right)} .
$$

A Fig. 3 traz um esboço da curva descrita pela equação (9), bem como as curvas que descrevem os campos internos e externos da casca. Observa-se, facilmente, que

$$
\lim _{r \rightarrow R_{1}} \frac{Q}{4 \pi \varepsilon_{\circ}} \frac{r}{R_{2}^{3}} \frac{\left(1-\frac{R_{1}{ }^{3}}{r^{3}}\right)}{\left(1-\frac{R_{1}{ }^{3}}{R_{2}^{3}}\right)}=0
$$

e, também,

$$
\lim _{r \rightarrow R_{2}} \frac{Q}{4 \pi \varepsilon_{\circ}} \frac{r}{R_{2}^{3}} \frac{\left(1-\frac{R_{1}^{3}}{r^{3}}\right)}{\left(1-\frac{R_{1}^{3}}{R_{2}^{3}}\right)}=\frac{1}{4 \pi \varepsilon_{\circ}} \frac{Q}{R_{2}^{2}},
$$

não havendo, portanto, descontinuidade nas interfaces interna e externa. Da mesma forma, no modelo hipotético assumido nesta seção, não há região não diferenciável na curva de potencial (Veja Fig. 3.

Além do modelo resultar em uma curva contínua para o campo e um potencial diferenciável para qualquer $r$, é fundamental que, por consistência lógica, no limite em que a espessura da casca tende a zero, obtenha-se os mesmos resultados de quando se assume uma distribuição singular de carga. De fato, escrevendo $R_{1}$ e $R_{2}$ como função de $\delta$ e avaliando o campo exatamente no meio da casca $(r=R)$, a equação 9 se resume a

$$
E=\frac{Q}{4 \pi \varepsilon_{\circ}} \frac{3 R^{2}-3 \delta R+\delta^{2}}{R^{2}\left(6 R^{2}+2 \delta^{2}\right)},
$$

de modo que

$$
\lim _{\delta \rightarrow 0} E(R)=\frac{1}{8 \pi \varepsilon_{\circ}} \frac{Q}{R^{2}}=\frac{\sigma}{2 \epsilon_{0}} .
$$

É importante ressaltar que, embora o limite 13 resulte de um modelo bastante rudimentar (distribuição uniforme) para distribuição das cargas no interior da camada de espessura $2 \delta$, o mesmo resultado pode ser obtido para qualquer que seja a forma com que as cargas

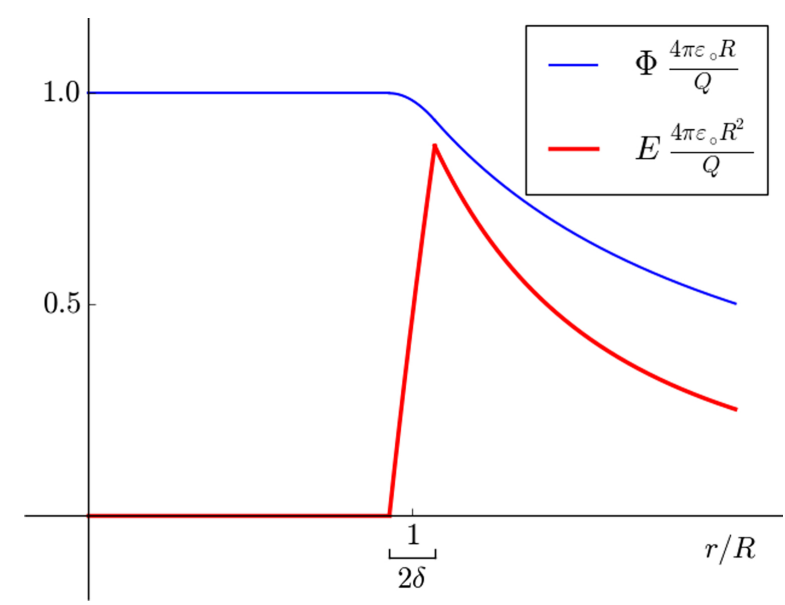

Figura 3: Campo elétrico e potencial elétrico devido a uma casca esférica de espessura $2 \delta$ e carga elétrica $Q$, uniformemente distribuída. 
se organizam nessa camada, conforme mostrado em 12 . Por outro lado, o uso do modelo de distribuição uniforme de cargas ao longo da casca, nesta seção, torna o problema didaticamente tratável em cursos básicos, sem gerar perda de generalidade no resultado final.

\section{Considerações finais}

É importante notar que, além de levar ao mesmo resultado matemático presente na literatura citada na Seç. 1 . a análise desenvolvida na Seç. 3 nos permite entendêlo como um limite do modelo e não como uma inconsistência entre o que é previsto na Teoria Eletromagnética $(\vec{E}(r)=-\nabla V(r), \forall r \in \Re)$ e o resultado que o modelo retorna (potencial não diferenciável em $r=R$ ). Além disso, o resultado limite apresentado na seção 3 independe do modo como o excesso de cargas se rearranja na superfície do condutor em uma espessura finita, conforme mostrado em 12. Nesse sentido, embora possamos obter o resultado da equação (5) de diferentes formas, quando se assume uma distribuição de cargas singular é preciso ter clareza que o campo não estará necessariamente definido em todos os pontos, não fazendo sentido expressar um valor para $\vec{E}(R)$. Dessa forma, tal como descreve [3], também entendemos que o esboço gráfico apresentado por [10] e por [2] (aqui reproduzido na Seç. 2) levam a uma compreensão equivocada do problema, em especial quando os detalhes do modelo não são claramente explicitados.

\section{Agradecimentos}

Agradecemos aos revisores deste artigo pelas contribuições no sentido de tornar nossas afirmações mais precisas e pelas referências sugeridas. Agradecemos também ao professor Fabiano Pereira pelos diálogos balizados por Análise Matemática.

\section{Referências}

[1] J. Machado e S.M. Silva Corrêa de Souza Cruz, Ciência e Educação 17, 887 (2011).

[2] G. Salvatore, Revista Brasileira de Ensino de Física 30, 1701 (2008).

[3] G.E. Assad, Revista Brasileira de Ensino de Física 34, 4701 (2012).

[4] D. Halliday, R. Resnick e J. Walker, Fundamentos de Física, Volume 3: Eletromagnetismo (LTD, Rio de Janeiro, 2012), 9 a ed, p. 92-93.

[5] H.M. Nussenzveig, Curso de Física Básica 3 - Eletromagnetismo, Volume 3 (Blücher, São Paulo, 1997), $1^{\text {a }}$ ed, p. 31-32.

[6] A. Máximo e B. Alvarenga, Física, Volume 3 (Scipione, São Paulo, 2008), $1^{\mathrm{a}}$ ed, p. 61-62.

[7] C. Xavier e B. Barreto, Física Aula por Aula, Volume 3 (FTD, São Paulo, 2010), 1aㅡ ed, p. 78-79.
[8] J.R. Bonjorno, R.A. Bonjorno, V. Bonjorno e C.M. Ramos, Física: História e Cotidiano, Volume 3 (FTD, São Paulo, 2013), $2^{\text {a }}$ ed, p. 47-49.

[9] R.P. Feynman; R.B. Leighton e M. Sands (orgs.), in: Lições de Física de Feynman, Volume II (Bookman, Porto Alegre, 2008), edição definitiva, cap. Aplicações da Lei de Gauss: o campo de um condutor (paginação irregular).

[10] F. Ramalho Junior, N.G. Ferraro e P.A de T. Soares, Fundamentos da Física, Volume 3 (Moderna, São Paulo, 2004), 8 $8^{\mathrm{a}}$ ed, p. 75-76.

[11] J.D. Jackson, Classical Electrodynamics (Wiley, New York, 1998), third edition, p. 20-21.

[12] E.M. Purcell, Berkeley Physics Course, Volumen 2: Electricidad y Magnetismo (Editorial Reverté, Barcelona, 1988), $2^{\mathrm{a}}$ ed, p. 1-39. 